Proyecciones Journal of Mathematics

Vol. 36, Nº 3, pp. 461-484, September 2017.

Universidad Católica del Norte

Antofagasta - Chile

\title{
Hyperstability of cubic functional equation in ultrametric spaces
}

\author{
Youssef Aribou \\ University of Ibn Tofail, Morocco \\ Muaadh Almahalebi \\ University of Ibn Tofail, Morocco \\ and \\ Samir kabbaj \\ University of Ibn Tofail, Morocco \\ Received : December 2016. Accepted : March 2017
}

\begin{abstract}
In this paper, we present the hyperstability results of cubic functional equations in ultrametric Banach spaces.
\end{abstract}

Keywords : Stability, hyperstability, ultrametric space, cubic functional equation.

Mathematics Subject Classification : Primary 39B82; Secondary 39B52, $47 \mathrm{H} 10$. 


\section{Introduction}

The starting point of studying the stability of functional equations seems to be the famous talk of Ulam [29] in 1940, in which he discussed a number of important unsolved problems. Among those was the question concerning the stability of group homomorphisms.

Let $G_{1}$ be a group and let $G_{2}$ be a metric group with a metric d(.,.). Given $\varepsilon>0$, does there exists a $\delta>0$ such that if a mapping $h: G_{1} \rightarrow G_{2}$ satisfies the inequality $d(h(x y), h(x) h(y))<\delta$ for all $x, y \in G_{1}$, then there exists a homomorphism $H: G_{1} \rightarrow G_{2}$ with $d(h(x), H(x))<\varepsilon$ for all $x \in G_{1}$.

The first partial answer, in the case of Cauchy equation in Banach spaces, to Ulam question was given by Hyers [22]. Later, the result of $\mathrm{Hy}-$ ers was first generalized by Aoki [?]. And only much later by Rassias [27] and Găvruţa [20]. Since then, the stability problems of several functional equations have been extensively investigated.

We say a functional equation is hyperstable if any function $f$ satisfying the equation approximately (in some sense) must be actually a solution to it. It seems that the first hyperstability result was published in [11] and concerned the ring homomorphisms. However, the term hyperstability has been used for the first time in [25]. Quite often the hyperstability is confused with superstability, which admits also bounded functions. Numerous papers on this subject have been published and we refer to [1]-[5], [8], [15][18], [21], [25], [26], [28].

Throughout this paper, $\mathbf{N}$ stands for the set of all positive integers, $\mathbf{N}_{0}:=\mathbf{N} \cup\{0\}, N_{m_{0}}$ the set of integers $\geq m_{0}, \mathbf{R}_{+}:=[0, \infty)$ and we use the notation $X_{0}$ for the set $X \backslash\{0\}$.

Let us recall (see, for instance, [24] some basic definitions and facts concerning non-Archimedean normed spaces.

Definition 1.1. By a non-Archimedean field we mean a field $\mathbf{K}$ equipped with a function (valuation) $|\cdot|: \mathbf{K} \rightarrow[0, \infty)$ such that for all $r, s \in \mathbf{K}$, the following conditions hold:

1. $|r|=0$ if and only if $r=0$, 
2. $|r s|=|r||s|$,

3. $|r+s| \leq \max \{|r|,|s|\}$.

The pair $(\mathbf{K},||$.$) is called a valued field.$

In any non-Archimedean field we have $|1|=|-1|=1$ and $|n| \leq 1$ for $n \in \mathbf{N}_{0}$. In any field $\mathbf{K}$ the function $|\cdot|: \mathbf{K} \rightarrow \mathbf{R}_{+}$given by

$$
|x|:= \begin{cases}0, & x=0, \\ 1, & x \neq 0,\end{cases}
$$

is a valuation which is called trivial, but the most important examples of non-Archimedean fields are $p$-adic numbers which have gained the interest of physicists for their research in some problems coming from quantum physics, $p$-adic strings and superstrings.

Definition 1.2. Let $X$ be a vector space over a scalar field $\mathbf{K}$ with a nonArchimedean non-trivial valuation $|\cdot|$. A function $\|\cdot\|_{*}: X \rightarrow \mathbf{R}$ is a non-Archimedean norm (valuation) if it satisfies the following conditions:

1. $\|x\|_{*}=0$ if and only if $x=0$,

2. $\|r x\|_{*}=|r|\|x\|_{*}(r \in \mathbf{K}, x \in X)$,

3. The strong triangle inequality (ultrametric); namely

$$
\|x+y\|_{*} \leq \max \left\{\|x\|_{*},\|y\|_{*}\right\} \quad x, y \in X .
$$

Then $\left(X,\|\cdot\|_{*}\right)$ is called a non-Archimedean normed space or an ultrametric normed space.

Definition 1.3. Let $\left\{x_{n}\right\}$ be a sequence in a non-Archimedean normed space $X$.

1. A sequence $\left\{x_{n}\right\}_{n=1}^{\infty}$ in a non-Archimedean space is a Cauchy sequence iff the sequence $\left\{x_{n+1}-x_{n}\right\}_{n=1}^{\infty}$ converges to zero;

2. The sequence $\left\{x_{n}\right\}$ is said to be convergent if, there exists $x \in X$ such that, for any $\varepsilon>0$, there is a positive integer $N$ such that $\left\|x_{n}-x\right\|_{*} \leq \varepsilon$, for all $n \geq N$. Then the point $x \in X$ is called the limit of the sequence $\left\{x_{n}\right\}$, which is denoted by $\lim _{n \rightarrow \infty} x_{n}=x$; 
3. If every Cauchy sequence in $X$ converges, then the non-Archimedean normed space $X$ is called a non-Archimedean Banach space or an ultrametric Banach space.

Let $X, Y$ be normed spaces. A function $f: X \rightarrow Y$ is Cubic provided it satisfies the functional equation

$f(2 x+y)+f(2 x-y)=2 f(x+y)+2 f(x-y)+12 f(x)$ for all $x, y \in X$,

and we can say that $f: X \rightarrow Y$ is Cubic on $X_{0}$ if it satisfies (1.1) for all $x, y \in X_{0}$ such that $x+y \neq 0$.

In 2013, A. Bahyrycz and al. [7] used the fixed point theorem from [12, Theorem 1] to prove the stability results for a generalization of $p$-Wright affine equation in ultrametric spaces. Recently, corresponding results for more general functional equations (in classical spaces) have been proved in [9], [10], [30] and [31].

In this paper, by using the fixed point method derived from [8], [15] and [14], we present some hyperstability results for the equation (1.1) in ultrametric Banach spaces. Before proceeding to the main results, we state Theorem 1.4 which is useful for our purpose. To present it, we introduce the following three hypotheses:

(H1) $X$ is a nonempty set, $Y$ is an ultrametric Banach space over a nonArchimedean field, $f_{1}, \ldots, f_{k}: X \longrightarrow X$ and $L_{1}, \ldots, L_{k}: X \longrightarrow \mathbf{R}_{+}$ are given.

(H2) $\mathcal{T}: Y^{X} \longrightarrow Y^{X}$ is an operator satisfying the inequality

$$
\begin{gathered}
\|\mathcal{T} \xi(x)-\mathcal{T} \mu(x)\|_{*} \leq \max _{1 \leq i \leq k}\left\{L_{i}(x)\left\|\xi\left(f_{i}(x)\right)-\mu\left(f_{i}(x)\right)\right\|_{*}\right\}, \\
\xi, \mu \in Y^{X}, \quad x \in X .
\end{gathered}
$$

(H3) $\Lambda: \mathbf{R}_{+}^{X} \longrightarrow \mathbf{R}_{+}^{X}$ is a linear operator defined by

$$
\Lambda \delta(x):=\max _{1 \leq i \leq k}\left\{L_{i}(x) \delta\left(f_{i}(x)\right)\right\}, \quad \delta \in \mathbf{R}_{+}^{X}, \quad x \in X .
$$


Thanks to a result due to J. Brzdęk and K. Ciepliñski [13, Remark 2], we state a slightly modified version of the fixed point theorem [12, Theorem 1] in ultrametric spaces. We use it to assert the existence of a unique fixed point of operator $\mathcal{T}: Y^{X} \longrightarrow Y^{X}$.

Theorem 1.4. Let hypotheses (H1)-(H3) be valid and functions $\varepsilon: X \longrightarrow$ $\mathbf{R}_{+}$and $\varphi: X \longrightarrow Y$ fulfil the following two conditions

$$
\begin{gathered}
\|\mathcal{T} \varphi(x)-\varphi(x)\|_{*} \leq \varepsilon(x), \quad x \in X, \\
\lim _{n \rightarrow \infty} \Lambda^{n} \varepsilon(x)=0, \quad x \in X .
\end{gathered}
$$

Then there exists a unique fixed point $\psi \in Y^{X}$ of $\mathcal{T}$ with

$$
\|\varphi(x)-\psi(x)\|_{*} \leq \sup _{n \in \mathbf{N}_{0}} \Lambda^{n} \varepsilon(x), \quad x \in X .
$$

Moreover

$$
\psi(x):=\lim _{n \rightarrow \infty} \mathcal{T}^{n} \varphi(x), \quad x \in X
$$

\section{Main results}

In this section, using Theorem 1.4 as a basic tool to prove the hyperstability results of the cubic functional equation in ultrametric Banach spaces.

Theorem 2.1. Let $(X,\|\cdot\|)$ and $\left(Y,\|\cdot\|_{*}\right)$ be normed space and ultrametric Banach space respectively, $c \geq 0, p, q \in \mathbf{R}, p+q<0$ and let $f: X \rightarrow Y$ satisfy

$\|f(2 x+y)+f(2 x-y)-2 f(x+y)-2 f(x-y)-12 f(x)\|_{*} \leq c\|x\|^{p}\|y\|^{q}$, (2.1)

for all $x, y \in X_{0}$. Then $f$ is cubic on $X_{0}$.

Proof. Take $m \in \mathbf{N}$ such that

$$
\alpha_{m}:=\left(\frac{m-1}{2}\right)^{p+q}<1 \text { and } m \geq 4
$$

Since $p+q<0$, one of $p, q$ must be negative. Assume that $q<0$ and replace $y$ by $m x$ and $x$ by $\frac{m+1}{2} x$ in (2.1). Thus 


$$
\begin{gathered}
\left\|12 f\left(\frac{m+1}{2} x\right)+2 f\left(\frac{3 m+1}{2} x\right)+2 f\left(\frac{1-m}{2} x\right)-f((2 m+1) x)-f(x)\right\|_{*} \\
\leq c m^{q}\left(\frac{m+1}{2}\right)^{p}\|x\|^{p+q},
\end{gathered}
$$

Define operators $\mathcal{T}_{m}: Y^{X_{0}} \rightarrow Y^{X_{0}}$ and $\Lambda_{m}: \mathbf{R}_{+}^{X_{0}} \rightarrow \mathbf{R}_{+}^{X_{0}}$ by

$$
\mathcal{T}_{m} \xi(x):=12 \xi\left(\left(\frac{m+1}{2}\right) x\right)+2 \xi\left(\left(\frac{3 m+1}{2}\right) x\right)+2 \xi\left(\left(\frac{1-m}{2}\right) x\right)
$$

$$
-\xi((2 m+1) x), \quad \xi \in Y^{X_{0}}, x \in X_{0},
$$

$\Lambda_{m} \delta(x):=\max \left\{\delta\left(\left(\frac{m+1}{2}\right) x\right), \delta\left(\left(\frac{3 m+1}{2}\right) x\right), \delta\left(\left(\frac{1-m}{2}\right) x\right)\right.$

$$
\delta(2 m+1), \quad \delta \in \mathbf{R}_{+}^{X_{0}}, x \in X_{0}
$$

and write

$$
\varepsilon_{m}(x):=c m^{q}\left(\frac{m+1}{2}\right)^{p}\|x\|^{p+q}, \quad x \in X_{0} .
$$

It is easily seen that $\Lambda_{m}$ has the form described in (H3) with $k=4$, $f_{1}(x)=\left(\frac{m+1}{2}\right) x, f_{2}(x)=\left(\frac{3 m+1}{2}\right) x, f_{3}(x)=\left(\frac{1-m}{2}\right) x, f_{4}(x)=(2 m+1) x$ and $L_{1}(x)=L_{2}(x)=L_{3}(x)=L_{4}(x)=1$. Further, (2.1) can be written in the following way

$$
\left\|\mathcal{T}_{m} f(x)-f(x)\right\|_{*} \leq \varepsilon_{m}(x), \quad x \in X_{0} .
$$

Moreover, for every $\xi, \mu \in Y^{X_{0}}, x \in X_{0}$

$$
\begin{gathered}
\left\|\mathcal{T}_{m} \xi(x)-\mathcal{T}_{m} \mu(x)\right\|_{*}=\| 12 \xi\left(\left(\frac{m+1}{2}\right) x\right)+2 \xi\left(\left(\frac{3 m+1}{2}\right) x\right)+2 \xi\left(\left(\frac{1-m}{2}\right) x\right) \\
-\xi((2 m+1) x)-12 \mu\left(\left(\frac{m+1}{2}\right) x\right)-2 \mu\left(\left(\frac{3 m+1}{2}\right) x\right)-2 \mu\left(\left(\frac{1-m}{2}\right) x\right) \\
+\xi((2 m+1) x) \|_{*} \\
\leq \max \left\{\begin{array}{l}
\left\|\xi\left(\left(\frac{m+1}{2}\right) x\right)-\mu\left(\left(\frac{m+1}{2}\right) x\right)\right\|_{*},\left\|\xi\left(\frac{3 m+1}{2} x\right)-\mu\left(\frac{3 m+1}{2} x\right)\right\|_{*}, \\
\left.\left.\left\|\xi\left(\frac{1-m}{2} x\right)-\mu\left(\frac{1-m}{2} x\right)\right\|_{*}, \| \xi(2 m+1) x\right)-\mu((2 m+1) x) \|_{*}\right\} .
\end{array}\right.
\end{gathered}
$$


So, (H2) is valid.

By using mathematical induction, we will show that for each $x \in X_{0}$ we have

$$
\Lambda_{m}^{n} \varepsilon_{m}(x)=c m^{q}\left(\frac{m+1}{2}\right)^{p}\|x\|^{p+q} \alpha_{m}^{n}
$$

where $\alpha_{m}=\left(\frac{m-1}{2}\right)^{p+q}$. From (2.4), we obtain that (2.5) holds for $n=0$. Next, we will assume that (2.5) holds for $n=k$, where $k \in \mathbf{N}$. Then we have

$$
\begin{gathered}
\Lambda_{m}^{k+1} \varepsilon_{m}(x)=\Lambda_{m}\left(\Lambda_{m}^{k} \varepsilon_{m}(x)\right)=\max \left\{\Lambda_{m}^{k} \varepsilon_{m}\left(\left(\frac{m+1}{2}\right) x\right), \Lambda_{m}^{k} \varepsilon_{m}\left(\left(\frac{3 m+1}{2}\right) x\right),\right. \\
\Lambda_{m}^{k} \varepsilon_{m}\left(\left(\frac{m-1}{2}\right) x\right), \Lambda_{m}^{k} \varepsilon_{m}((2 m+1) x) \\
=\max \left\{c m^{q}\left(\frac{m+1}{2}\right)^{p}\|x\|^{p+q} \alpha_{m}^{k}\left(\frac{m+1}{2}\right)^{p+q}, c m^{q}\left(\frac{m+1}{2}\right)^{p}\|x\|^{p+q} \alpha_{m}^{k}\left(\frac{3 m+1}{2}\right)^{p+q},\right. \\
c m^{q}\left(\frac{m+1}{2}\right)^{p}\|x\|^{p+q} \alpha_{m}^{k}\left(\frac{m-1}{2}\right)^{p+q} c m^{q}\|x\|^{p+q} \alpha_{m}^{k}(2 m+1)^{p+q} \\
=c m^{q}\|x\|^{p+q} \alpha_{m}^{k} \max \left\{\left(\frac{m+1}{2}\right)^{p+q},\left(\frac{3 m+1}{2}\right)^{p+q},\left(\frac{m-1}{2}\right)^{p+q},(2 m+1)^{p+q}\right\} \\
=c m^{q}\left(\frac{m+1}{2}\right)^{p}\|x\|^{p+q} \alpha_{m}^{k+1}, \quad x \in X_{0} .
\end{gathered}
$$

This shows that (2.5) holds for $n=k+1$. Now we can conclude that the inequality (2.5) holds for all $n \in \mathbf{N}_{0}$. From (2.5), we obtain

$$
\lim _{n \rightarrow \infty} \Lambda^{n} \varepsilon_{m}(x)=0,
$$

for all $x \in X_{0}$. Hence, according to Theorem 1.4, there exists a unique solution $C_{m}: X_{0} \rightarrow Y$ of the equation

$C_{m}(x)=12 C_{m}\left(\left(\frac{m+1}{2}\right) x\right)+2 C_{m}\left(\left(\frac{3 m+1}{2}\right) x\right)+2 C_{m}\left(\left(\frac{1-m}{2}\right) x\right)$

$$
\begin{gathered}
-C_{m}((2 m+1) x), \\
x \in X_{0}
\end{gathered}
$$

such that

$(2.8)\left\|f(x)-C_{m}(x)\right\|_{*} \leq \sup _{n \in \mathbf{N}_{0}}\left\{c m^{q}\left(\frac{m+1}{2}\right)^{p}\|x\|^{p+q} \alpha_{m}^{n}\right\}, \quad x \in X_{0}$. 
Moreover,

$$
C_{m}(x):=\lim _{n \rightarrow \infty} \mathcal{T}_{m}^{n} f(x)
$$

for all $x \in X_{0}$. Now we show that

$\left\|\mathcal{T}_{m}^{n} f(2 x+y)+\mathcal{T}_{m}^{n} f(2 x-y)-2 \mathcal{T}_{m}^{n} f(x+y)-\mathcal{T}_{m}^{n} f(x-y)-12 \mathcal{T}_{m}^{n} f(x)\right\|_{*}$

$$
\leq c \alpha_{m}^{n}\|x\|^{p}\|y\|^{q}
$$

for every $x, y \in X_{0}$ such that $x+y \neq 0$. Since the case $n=0$ is just (2.1), take $k \in \mathbf{N}$ and assume that (2.9) holds for $n=k$ and every $x, y \in X_{0}$ such that $x+y \neq 0$. Then

$$
\begin{aligned}
& \left\|\mathcal{T}_{m}^{k+1} f(2 x+y)+\mathcal{T}_{m}^{k+1} f(2 x-y)-2 \mathcal{T}_{m}^{k+1} f(x+y)-2 \mathcal{T}_{m}^{k+1} f(x-y)-12 \mathcal{T}_{m}^{k+1} f(x)\right\|_{*} \\
& =\| 12 \mathrm{~T}_{m}^{k} f\left(\left(\frac{m+1}{2}\right)(2 x+y)\right)+2 \mathcal{T}_{m}^{k} f\left(\left(\frac{3 m+1}{2}\right)(2 x+y)\right)+2 \mathcal{T}_{m}^{k} f\left(\left(\frac{1-m}{2}\right)(2 x+y)\right) \\
& -\mathrm{T}_{m}^{k} f((2 m+1)(2 x+y))+12 \mathcal{T}_{m}^{k} f\left(\left(\frac{m+1}{2}\right)(2 x-y)\right)+2 \mathcal{T}_{m}^{k} f\left(\left(\frac{3 m+1}{2}\right)(2 x-y)\right) \\
& +2 \mathrm{~T}_{m}^{k} f\left(\left(\frac{1-m}{2}\right)(2 x-y)\right)-\mathcal{T}_{m}^{k} f((2 m+1)(2 x-y))-24 \mathcal{T}_{m}^{k} f\left(\left(\frac{m+1}{2}\right)(x+y)\right) \\
& \quad-4 \mathrm{~T}_{m}^{k} f\left(\left(\frac{3 m+1}{2}\right)(x+y)\right)-4 \mathcal{T}_{m}^{k} f\left(\left(\frac{1-m}{2}\right)(x+y)\right)+2 \mathcal{T}_{m}^{k} f((2 m+1)(x+y)) \\
& \quad-24 \mathrm{~T}_{m}^{k} f\left(\left(\frac{m+1}{2}\right)(x-y)\right)-4 \mathcal{T}_{m}^{k} f\left(\left(\frac{3 m+1}{2}\right)(x-y)\right)-4 \mathcal{T}_{m}^{k} f\left(\left(\frac{1-m}{2}\right)(x-y)\right) \\
& \quad+2 \mathcal{T}_{m}^{k} f((2 m+1)(x-y))-144 \mathcal{T}_{m}^{k} f\left(\left(\frac{m+1}{2}\right)(x)\right)-24 \mathcal{T}_{m}^{k} f\left(\left(\frac{3 m+1}{2}\right)(x)\right) \\
& \quad-24 \mathrm{~T}_{m}^{k} f\left(\left(\frac{1-m}{2}\right)(x)\right)+12 \mathcal{T}_{m}^{k} f((2 m+1)(x)) \|_{*}
\end{aligned}
$$




$$
\begin{aligned}
& \leq \max \left\{\| \mathcal{T}_{m}^{n} f\left(\frac{m+1}{2}(2 x+y)\right)+\mathcal{T}_{m}^{n} f\left(\frac{m+1}{2}(2 x-y)\right)-2 \mathcal{T}_{m}^{n} f\left(\frac{m+1}{2}(x+y)\right)\right. \\
& -\mathcal{T}_{m}^{n} f\left(\frac{m+1}{2}(x-y)\right)-12 \mathcal{T}_{m}^{n} f\left(\frac{m+1}{2} x\right) \|_{*} \\
& \| \mathcal{T}_{m}^{n} f\left(\frac{3 m+1}{2}(2 x+y)\right)+\mathcal{T}_{m}^{n} f\left(\frac{3 m+1}{2}(2 x-y)\right)-2 \mathcal{T}_{m}^{n} f\left(\frac{3 m+1}{2}(x+y)\right) \\
& -\mathcal{T}_{m}^{n} f\left(\frac{3 m+1}{2}(x-y)\right)-12 \mathcal{T}_{m}^{n} f\left(\frac{3 m+1}{2} x\right) \|_{*}, \\
& \| \mathcal{T}_{m}^{n} f\left(\frac{1-m}{2}(2 x+y)\right)+\mathcal{T}_{m}^{n} f\left(\frac{1-m}{2}(2 x-y)\right)-2 \mathcal{T}_{m}^{n} f\left(\frac{1-m}{2}(x+y)\right) \\
& -\mathcal{T}_{m}^{n} f\left(\frac{1-m}{2}(x-y)\right)-12 \mathcal{T}_{m}^{n} f\left(\frac{1-m}{2} x\right) \|_{*}, \\
& \| \mathcal{T}_{m}^{n} f((2 m+1)(2 x+y))+\mathcal{T}_{m}^{n} f((2 m+1)(2 x-y))-2 \mathcal{T}_{m}^{n} f((2 m+1)(x+y)) \\
& \left.-\mathcal{T}_{m}^{n} f((2 m+1)(x-y))-12 \mathcal{T}_{m}^{n} f((2 m+1) x) \|_{*}\right\} \\
& \leq \max \left\{c \alpha_{m}^{k}\|x\|^{p}\|y\|^{q}\left(\frac{m+1}{2}\right)^{p+q}, c \alpha_{m}^{k}\|x\|^{p}\|y\|^{q}\left(\frac{3 m+1}{2}\right)^{p+q},\right. \\
& \left.c \alpha_{m}^{k}\|x\|^{p}\|y\|^{q}\left(\frac{1-m}{2}\right)^{p+q}, c \alpha_{m}^{k}\|x\|^{p}\|y\|^{q}(2 m+1)^{p+q}\right\} \\
& =c \alpha_{m}^{k}\|x\|^{p}\|y\|^{q} \max \left\{\left(\frac{m+1}{2}\right)^{p+q},\left(\frac{3 m+1}{2}\right)^{p+q},\left(\frac{m-1}{2}\right)^{p+q},(3 m+1)^{p+q}\right\} \\
& \leq c \alpha_{m}^{k+1}\|x\|^{p}\|y\|^{q}
\end{aligned}
$$


for all $x, y \in X_{0}$ such that $x+y \neq 0$. Thus, by induction we have shown that (2.9) holds for every $n \in \mathbf{N}_{0}$. Letting $n \rightarrow \infty$ in (2.9), we obtain that

$$
C_{m}(2 x+y)+C_{m}(2 x-y)=2 C_{m}(x+y)+2 C_{m}(x-y)+12 C_{m}(x)
$$

for all $x, y \in X_{0}$ such that $x+y \neq 0$. In this way we obtain a sequence $\left\{C_{m}\right\}_{m \geq m_{0}}$ of cubic functions on $X_{0}$ such that

$$
\left\|f(x)-C_{m}(x)\right\|_{*} \leq \sup _{n \in \mathbf{N}_{0}}\left\{c m^{q}\left(\frac{m+1}{2}\right)^{p}\|x\|^{p+q} \alpha_{m}^{n}\right\}, \quad x_{0},
$$

this implies that

$$
\left\|f(x)-C_{m}(x)\right\|_{*} \leq c m^{q}\left(\frac{m+1}{2}\right)^{p}\|x\|^{p+q}, \quad x \in X_{0},
$$

It follows, with $m \rightarrow \infty$, that $f$ is Cubic on $X_{0}$.

In a similar way we can prove the following theorem.

Theorem 2.2. Let $(X,\|\cdot\|)$ and $\left(Y,\|\cdot\|_{*}\right)$ be normed space and ultrametric Banach space respectively, $c \geq 0, p, q \in \mathbf{R}, p+q>0$ and let $f: X \rightarrow Y$ satisfy

$\|f(2 x+y)+f(2 x-y)-2 f(x+y)-2 f(x-y)-12 f(x)\|_{*} \leq c\|x\|^{p}\|y\|^{q}$, (2.12)

for all $x, y \in X_{0}$. Then $f$ is Cubic on $X_{0}$.

Proof. Take $m \in \mathbf{N}$ such that

$$
\alpha_{m}=\left(\frac{m-4}{m}\right)^{p+q}<1 \text { and } 6<m .
$$

Since $p+q>0$, one of $p, q$ must be positive; let $q>0$ and replace $y$ by $\frac{-2}{m} y$ and $x$ by $\frac{m-2}{2 m} y$ in (2.12). Thus

$$
\begin{gathered}
\left\|12 f\left(\frac{m-2}{2 m} y\right)+2 f\left(\frac{m+2}{2 m} y\right)+2 f\left(\frac{m-6}{2 m} y\right)-f\left(\frac{m-4}{m} y\right)-f(y)\right\|_{*} \\
\leq c\left(\frac{2}{m}\right)^{q}\left(\frac{m-2}{2 m}\right)^{p}\|y\|^{p+q}, \quad x \in X_{0} .
\end{gathered}
$$


Write

$$
\begin{aligned}
\mathcal{T}_{m} \xi(x) & :=12 \xi\left(\left(\frac{m-2}{2 m}\right) x\right)+2 \xi\left(\left(\frac{m+2}{2 m}\right) x\right)+2 \xi\left(\left(\frac{m-6}{2 m}\right) x\right)-\xi\left(\left(\frac{m-4}{m}\right) x\right), \\
& \xi \in Y^{X_{0}}, x \in X_{0},
\end{aligned}
$$

and

$$
\varepsilon_{m}(x):=c\left(\frac{2}{m}\right)^{q}\left(\frac{m-1}{2 m}\right)^{p}\|x\|^{p+q}, \quad x \in X_{0},
$$

then (2.13) takes form

$$
\left\|\mathcal{T}_{m} f(x)-f(x)\right\|_{*} \leq \varepsilon_{m}(x), \quad x \in X_{0} .
$$

Define

$$
\begin{aligned}
& \Lambda_{m} \delta(x):= \max \left\{\delta\left(\left(\frac{m-2}{2 m}\right) x\right), \delta\left(\left(\frac{m+2}{2 m}\right) x\right), \delta\left(\left(\frac{m-6}{2 m}\right) x\right), \delta\left(\left(\frac{m-4}{m}\right) x\right)\right\}, \\
& \delta \in \mathbf{R}_{+}^{X_{0}}, x \in X_{0} .
\end{aligned}
$$

Then it is easily seen that $\Lambda_{m}$ has the form described in (H3) with $k=4$, $f_{1}(x)=\left(\frac{m-2}{2 m}\right) x, f_{2}(x)=\left(\frac{m+2}{2 m}\right) x, f_{3}(x)=\left(\frac{m-6}{2 m}\right) x, f_{2}(x)=\left(\frac{m-4}{m}\right) x$ and $L_{1}(x)=1, L_{2}(x)=1, L_{3}(x)=1, L_{4}(x)=1$.

Moreover, for every $\xi, \mu \in Y^{X_{0}}, x \in X_{0}$

$$
\begin{array}{r}
\left\|\mathcal{T}_{m} \xi(x)-\mathcal{T}_{m} \mu(x)\right\|_{*}=\| 12 \xi\left(\left(\frac{m-2}{2 m}\right) x\right)+2 \xi\left(\left(\frac{m+2}{2 m}\right) x\right)+2 \xi\left(\left(\frac{m-6}{2 m}\right) x\right) \\
-\xi\left(\left(\frac{m-4}{m}\right) x\right)-12 \mu\left(\left(\frac{m-2}{2 m}\right) x\right)-2 \mu\left(\left(\frac{m+2}{2 m}\right) x\right)-2 \mu\left(\left(\frac{m-6}{2 m}\right) x\right) \\
+\xi\left(\left(\frac{m-4}{m}\right) x\right) \|_{*} \\
\leq \max \left\{\left\|\xi\left(\left(\frac{m-2}{2 m}\right) x\right)-\mu\left(\left(\frac{m-2}{2 m}\right) x\right)\right\|_{*},\left\|\xi\left(\frac{m+2}{2 m} x\right)-\mu\left(\frac{m+1}{2 m} x\right)\right\|_{*}\right. \\
\left.\left.\left\|\xi\left(\frac{m-6}{2 m} x\right)-\mu\left(\frac{m-6}{2 m} x\right)\right\|_{*}, \| \xi\left(\frac{m-4}{2 m}\right) x\right)-\mu\left(\left(\frac{m-4}{2 m}\right) x\right) \|_{*}\right\} .
\end{array}
$$

So, (H2) is valid.

By using mathematical induction, we will show that for each $x \in X_{0}$ we have

$$
\Lambda_{m}^{n} \varepsilon_{m}(x)=c\left(\frac{2}{m}\right)^{q}\left(\frac{m-2}{2 m}\right)^{p}\|x\|^{p+q} \alpha_{m}^{n}
$$


where $\alpha_{m}=\left(\frac{m-4}{m}\right)^{p+q}$. From (2.15), we obtain that (2.17) holds for $n=0$. Next, we will assume that (2.17) holds for $n=k$, where $k \in \mathbf{N}$. Then we have

$$
\begin{aligned}
& \Lambda_{m}^{k+1} \varepsilon_{m}(x)=\Lambda_{m}\left(\Lambda_{m}^{k} \varepsilon_{m}(x)\right)=\max \left\{\Lambda_{m}^{k} \varepsilon_{m}\left(\left(\frac{m-2}{2 m}\right) x\right), \Lambda_{m}^{k} \varepsilon_{m}\left(\left(\frac{m+2}{2 m}\right) x\right)\right. \\
& =c\left(\frac{2}{m}\right)^{q}\left(\frac{m-2}{2 m}\right)^{p}\|x\|^{p+q} \alpha_{m}^{k} \max \left\{\left(\frac{m-2}{2 m}\right)^{p+q},\left(\frac{m+2}{2 m}\right)^{p+q},\left(\frac{m-6}{2 m}\right)^{p+q},\left(\frac{m-4}{m}\right)^{p+q}\right\} \\
& =c\left(\frac{2}{m}\right)^{q}\left(\frac{m-2}{2 m}\right)^{p}\|x\|^{p+q} \alpha_{m}^{k+1}, \quad x \in X_{0} .
\end{aligned}
$$

This shows that (2.17) holds for $n=k+1$. Now we can conclude that the inequality (2.17) holds for all $n \in \mathbf{N}_{0}$. From (2.17), we obtain

$$
\lim _{n \rightarrow \infty} \Lambda^{n} \varepsilon_{m}(x)=0
$$

for all $x \in X_{0}$. Hence, according to Theorem 1.4, there exists a unique solution $C_{m}: X_{0} \rightarrow Y$ of the equation

$$
\begin{aligned}
& C_{m}(x)=12 C_{m}\left(\left(\frac{m-2}{2 m}\right) x\right)+2 C_{m}\left(\left(\frac{m+2}{2 m}\right) x\right)+2 C_{m}\left(\left(\frac{m-6}{2 m}\right) x\right) \\
& \quad-C_{m}\left(\left(\frac{m-4}{m}\right) x\right), x \in X_{0}
\end{aligned}
$$

such that

$$
\left\|f(x)-C_{m}(x)\right\|_{*} \leq \sup _{n \in \mathbf{N}_{0}}\left\{c\left(\frac{2}{m}\right)^{q}\left(\frac{m-2}{2 m}\right)^{p}\|x\|^{p+q} \alpha_{m}^{n}\right\}, \quad x_{0} .
$$

Moreover,

$$
C_{m}(x):=\lim _{n \rightarrow \infty} \mathcal{T}_{m}^{n} f(x)
$$

for all $x \in X_{0}$. We show that 
$\left\|\mathcal{T}_{m}^{n} f(2 x+y)+\mathcal{T}_{m}^{n} f(2 x-y)-2 \mathcal{T}_{m}^{n} f(x+y)-\mathcal{T}_{m}^{n} f(x-y)-12 \mathcal{T}_{m}^{n} f(x)\right\|_{*}$

$$
\leq c \alpha_{m}^{n}\|x\|^{p}\|y\|^{q}
$$

for every $x, y \in X_{0}$ such that $x+y \neq 0$. Since the case $n=0$ is just (2.12), take $k \in \mathbf{N}$ and assume that (2.20) holds for $n=k$ and every $x, y \in X_{0}$ such that $x+y \neq 0$. Then

$$
\begin{array}{rl}
\| \mathcal{T}_{m}^{k+1} & f(2 x+y)+\mathcal{T}_{m}^{k+1} f(2 x-y)-2 \mathcal{T}_{m}^{k+1} f(x+y)-2 \mathcal{T}_{m}^{k+1} f(x-y)-12 \mathcal{T}_{m}^{k+1} f(x) \|_{*} \\
\|= & 12 \mathcal{T}_{m}^{k} f\left(\left(\frac{m-2}{2 m}\right)(2 x+y)\right)+2 \mathcal{T}_{m}^{k} f\left(\left(\frac{m+2}{2 m}\right)(2 x+y)\right)+2 \mathcal{T}_{m}^{k} f\left(\left(\frac{m-6}{2 m}\right)(2 x+y)\right) \\
& -\mathcal{T}_{m}^{k} f\left(\left(\frac{m-4}{m}\right)(2 x+y)\right)+12 \mathcal{T}_{m}^{k} f\left(\left(\frac{m-2}{2 m}\right)(2 x-y)\right)+2 \mathcal{T}_{m}^{k} f\left(\left(\frac{m+2}{2 m}\right)(2 x-y)\right) \\
& +2 \mathcal{T}_{m}^{k} f\left(\left(\frac{m-6}{2 m}\right)(2 x-y)\right)-\mathcal{T}_{m}^{k} f\left(\left(\frac{m-4}{m}\right)(2 x-y)\right)-24 \mathcal{T}_{m}^{k} f\left(\left(\frac{m-2}{2 m}\right)(x+y)\right) \\
& -4 \mathcal{T}_{m}^{k} f\left(\left(\frac{m+2}{2 m}\right)(x+y)\right)-4 \mathcal{T}_{m}^{k} f\left(\left(\frac{m-6}{2 m}\right)(x+y)\right)+2 \mathcal{T}_{m}^{k} f\left(\left(\frac{m-4}{m}\right)(x+y)\right) \\
& -24 \mathcal{T}_{m}^{k} f\left(\left(\frac{m-2}{2 m}\right)(x-y)\right)-4 \mathcal{T}_{m}^{k} f\left(\left(\frac{m+2}{2 m}\right)(x-y)\right)-4 \mathcal{T}_{m}^{k} f\left(\left(\frac{m-6}{2 m}\right)(x-y)\right) \\
& +2 \mathcal{T}_{m}^{k} f\left(\left(\frac{m-4}{m}\right)(x-y)\right)-144 \mathcal{T}_{m}^{k} f\left(\left(\frac{m-2}{2 m}\right) x\right)-24 \mathcal{T}_{m}^{k} f\left(\left(\frac{m+2}{2 m}\right) x\right) \\
& -24 \mathcal{T}_{m}^{k} f\left(\left(\frac{m-6}{2 m}\right)(x)\right)+12 \mathcal{T}_{m}^{k} f\left(\left(\frac{m-4}{m}\right)(x)\right) \|_{*}
\end{array}
$$




$$
\begin{aligned}
\leq & \max \left\{\| \mathcal{T}_{m}^{n} f\left(\frac{m-2}{2 m}(2 x+y)\right)+\mathcal{T}_{m}^{n} f\left(\frac{m-2}{2 m}(2 x-y)\right)-2 \mathcal{T}_{m}^{n} f\left(\frac{m-2}{2 m}(x+y)\right)\right. \\
& -\mathcal{T}_{m}^{n} f\left(\frac{m-2}{2 m}(x-y)\right)-12 \mathcal{T}_{m}^{n} f\left(\frac{m-2}{2 m} x\right) \|_{*} \\
& \| \mathcal{T}_{m}^{n} f\left(\frac{m+2}{2 m}(2 x+y)\right)+\mathcal{T}_{m}^{n} f\left(\frac{m+2}{2 m}(2 x-y)\right)-2 \mathcal{T}_{m}^{n} f\left(\frac{m+2}{2 m}(x+y)\right) \\
& -\mathcal{T}_{m}^{n} f\left(\frac{m+2}{2 m}(x-y)\right)-12 \mathcal{T}_{m}^{n} f\left(\frac{m+2}{2 m} x\right) \|_{*}, \\
& \| \mathcal{T}_{m}^{n} f\left(\frac{m-6}{2 m}(2 x+y)\right)+\mathcal{T}_{m}^{n} f\left(\frac{m-6}{2 m}(2 x-y)\right)-2 \mathcal{T}_{m}^{n} f\left(\frac{m-6}{2 m}(x+y)\right) \\
& -\mathcal{T}_{m}^{n} f\left(\frac{m-6}{2 m}(x-y)\right)-12 \mathcal{T}_{m}^{n} f\left(\frac{m-6}{2 m} x\right) \|_{*} \\
& \left.c \alpha_{m}^{k}\|x\|_{m}^{p}\|y\|^{q}\left(\frac{m-6}{2 m}\right)^{p+q}, \alpha_{m}^{k}\|x\|^{p}\|y\|^{q}\left(\frac{m-4}{m}\right)^{p+q}\right\} \\
& \leq \alpha_{m}^{k} \|^{q} \max \left\{\left(\frac{m-2}{2 m}\right)^{p+q},\left(\frac{m+2}{2 m}\right)^{p+q},\left(\frac{m-6}{2 m}\right)^{p+q},\left(\frac{m-4}{m}\right)^{p+q}\right\} \\
& \| \mathcal{T}_{m}^{n} f\left(\left(\frac{m-4}{m}\right)(2 x+y)\right)+\mathcal{T}_{m}^{n} f\left(\left(\frac{m-4}{m}\right)(2 x-y)\right)-2 \mathcal{T}_{m}^{n} f\left(\left(\frac{m-4}{m}\right)(x+y)\right) \\
& \left.-\mathcal{T}_{m}^{n} f\left(\left(\frac{m-4}{m}\right)(x-y)\right)-12 \mathcal{T}_{m}^{n} f\left(\left(\frac{m-4}{m}\right) x\right) \|_{*}\right\}
\end{aligned}
$$


for all $x, y \in X_{0}$ such that $x+y \neq 0$. Thus, by induction we have shown that (2.20) holds for every $n \in \mathbf{N}_{0}$. Letting $n \rightarrow \infty$ in (2.20), we obtain that

$$
C_{m}(2 x+y)+C_{m}(2 x-y)=2 C_{m}(x+y)+2 C_{m}(x-y)+12 C_{m}(x)
$$

for all $x, y \in X_{0}$ such that $x+y \neq 0$. In this way we obtain a sequence $\left\{C_{m}\right\}_{m \geq m_{0}}$ of Cubic functions on $X_{0}$ such that

$$
\left\|f(x)-C_{m}(x)\right\|_{*} \leq \sup _{n \in \mathbf{N}_{0}}\left\{c\left(\frac{2}{m}\right)^{q}\left(\frac{m-2}{2 m}\right)^{p}\|x\|^{p+q} \alpha_{m}^{n}\right\}, \quad x_{0},
$$

this implies that

$$
\left\|f(x)-C_{m}(x)\right\|_{*} \leq c\left(\frac{2}{m}\right)^{q}\left(\frac{m-2}{2 m}\right)^{p} \mid x \|^{p+q}, \quad x \in X_{0} .
$$

It follows, with $m \rightarrow \infty$, that $f$ is Cubic on $X_{0}$.

It easy to show the hyperstability of Cubic equation on the set containing 0 . We present the following theorem and we refer to see $[8$, Theorem $5]$.

Theorem 2.3. Let $(X,\|\cdot\|)$ and $\left(Y,\|\cdot\|_{*}\right)$ be normed space and ultrametric Banach space respectively, $c \geq 0, p, q \in \mathbf{R}, p, q>0$ and let $f: X \rightarrow Y$ satisfy

$\| f(2 x+y))+f(2 x+y)-2 f(x+y)-2 f(x-y)-12 f(x)\left\|_{*} \leq c\right\| x\left\|^{p}\right\| y \|^{q}$, $(2.22)$

for all $x, y \in X_{0}$. Then $f$ is Cubic on $X_{0}$.

Proof. Same proof of last theorem

The above theorems imply in particular the following corollary, which shows their simple application.

Corollary 2.4. Let $(X,\|\cdot\|)$ and $\left(Y,\|\cdot\|_{*}\right)$ be normed space and ultrametric Banach space respectively, $G: X^{2} \rightarrow Y$ and $G(x, y) \neq 0$ for some $x, y \in X$ and

$$
\|G(x, y)\|_{*} \leq c\|x\|^{p}\|y\|^{q}, \quad x, y \in X
$$

where $c \geq 0, p, q \in \mathbf{R}$. Assume that the numbers $p, q$ satisfy one of the following conditions: 
1. $p+q<0$, and (2.1) holds for all $x, y \in X_{0}$,

2. $p+q>0$, and (2.12) holds for all $x, y \in X_{0}$.

Then the functional equation

$f(2 x+y)+f(2 x+y)=2 f(x+y)+2 f(x-y)+12 f(x)+G(x, y), \quad x, y \in X$

has no solution in the class of functions $g: X \rightarrow Y$.

In the following theorem, we present a general hyperstability for the Cubic equation where the control function is $\varphi(x)+\varphi(y)$, which corresponds to the approach introduced in [14]

Theorem 2.5. Let $(X,\|\cdot\|)$ be a normed space, $\left(Y,\|\cdot\|_{*}\right)$ be an ultrametric Banach space over a field $\mathbf{K}$, and $\varphi: X \rightarrow \mathbf{R}_{+}$be a function such that

$U:=\left\{n \in \mathbf{N}: \alpha_{n}:=\max \{\lambda(n), \lambda(3 n-1), \lambda(-n+1), \lambda(4 n-1)\}<1\right\}$ $(2.25)$

is an infinite set, where $\lambda(a):=\inf \left\{t \in \mathbf{R}_{+}: \varphi(a x) \leq t \varphi(x)\right.$ for all $\left.x \in X\right\}$ for all $a \in \mathbf{K}_{0}$. Suppose that

$$
\lim _{a \rightarrow \infty} \lambda(a)=0 \quad \text { and } \quad \lim _{a \rightarrow \infty} \lambda(-a)=0 .
$$

And $f: X \rightarrow Y$ satisfies the inequality

$\| f(2 x+y))+f(2 x+y)-2 f(x+y)-2 f(x-y)-12 f(x) \|_{*} \leq \varphi(x)+\varphi(y)$, $(2.26)$

for all $x, y \in X_{0}$. Then $f$ is Cubic on $X_{0}$.

Proof. Replacing $x$ by $m x$ and $y$ by $(-2 m+1) x$ for $m \in \mathbf{N}$ in $(2.27)$ we get

$$
\| f(x))+f((4 m-1) x)-2 f((-m+1) x)-2 f((3 m-1) x)-12 f(m x) \|_{*}
$$




$$
\leq \varphi((-2 m+1) x)+\varphi(m x)
$$

for all $x \in X_{0}$. For each $m \in U$, we define the operator $\mathcal{T}_{m}: Y^{X_{0}} \rightarrow Y^{X_{0}}$ by

$$
\begin{gathered}
\mathcal{T}_{m} \xi(x):=12 \xi(m x)+2 \xi((3 m-1) x)+2 \xi((-m+1) x)-\xi((4 m-1) x) \quad \xi \in Y^{X_{0}}, \\
x \in X_{0} .
\end{gathered}
$$

Further put

$\varepsilon_{m}(x):=\varphi((-2 m+1) x)+\varphi(m x) \leq(\lambda(-2 m+1)+\lambda(m x)) \varphi(x), \quad x \in X_{0}$.

Then the inequality (2.28) takes the form

$$
\left\|\mathcal{T}_{m} f(x)-f(x)\right\|_{*} \leq \varepsilon_{m}(x), \quad x \in X_{0} .
$$

For each $m \in U$, the operator $\Lambda_{m}: \mathbf{R}_{+}^{X_{0}} \rightarrow \mathbf{R}_{+}^{X_{0}}$ which is defined by

$$
\begin{aligned}
\Lambda_{m} \delta(x):= & \max \{\delta(m x), \delta((3 m-1) x)\}, \delta((-m+1) x), \\
& \delta((4 m-1) x), \quad \delta \in \mathbf{R}_{+}^{X_{0}}, x \in X_{0}
\end{aligned}
$$

has the form described in (H3) with $k=4$ and

$$
\begin{gathered}
f_{1}(x)=m x, \quad f_{2}(x)=(3 m-1) x, \quad f_{3}(x)=(-m+1) x, \quad f_{4}(x)=(4 m-1) x, \\
L_{1}(x)=L_{2}(x)=L_{2}(x)=L_{4}(x)=1
\end{gathered}
$$

for all $x \in X_{0}$. Moreover, for every $\xi, \mu \in Y^{X_{0}}, x \in X_{0}$

$$
\begin{aligned}
& \left\|\mathcal{T}_{m} \xi(x)-\mathcal{T}_{m} \mu(x)\right\|_{*}=\| 12 \xi((m) x)+2 \xi((3 m-1) x)+2 \xi((-m+1) x) \\
& -\xi((4 m-1) x)-12 \mu((m) x)-2 \mu((3 m-1) x)-2 \mu((-m+1) x) \\
& +\xi((4 m-1) x) \|_{*} \\
& \leq \max \left\{\|\xi((m) x)-\mu((m) x)\|_{*},\|\xi((3 m-1) x)-\mu((3 m-1) x)\|_{*},\right. \\
& \left.\left.\|\xi((-m+1) x)-\mu((-m+1) x)\|_{*}, \| \xi(4 m-1) x\right)-\mu((4 m-1) x) \|_{*}^{*}\right\} \text {. }
\end{aligned}
$$


So, (H2) is valid. By using mathematical induction, we will show that for each $x \in X_{0}$ we have

$$
\Lambda_{m}^{n} \varepsilon_{m}(x) \leq(\lambda(-2 m+1)+\lambda(m)) \alpha_{m}^{n} \varphi(x)
$$

From (2.29), we obtain that the inequality (2.30) holds for $n=0$. Next, we will assume that (2.30) holds for $n=k$, where $k \in \mathbf{N}$. Then we have

$$
\begin{aligned}
& \Lambda_{m}^{k+1} \varepsilon_{m}(x)=\Lambda_{m}\left(\Lambda_{m}^{k} \varepsilon_{m}(x)\right)=\max \left\{\Lambda_{m}^{k} \varepsilon_{m}(m x) ;, \Lambda_{m}^{k} \varepsilon_{m}((3 m-1) x),\right. \\
& \Lambda_{m}^{k} \varepsilon_{m}((-m+1) x), \Lambda_{m}^{k} \varepsilon_{m}((4 m-1) x) \\
& \leq(\lambda(m)+\lambda(-2 m+1)) \alpha_{m}^{k} \max \{\varphi(m x), \varphi((3 m-1) x), \\
& \varphi((-m+1) x), \varphi((4 m-1) x)\} \\
& \leq(\lambda(m)+\lambda(-2 m+1)) \alpha_{m}^{k+1} \varphi(x), \quad x \in X_{0} .
\end{aligned}
$$

This shows that (2.30) holds for $n=k+1$. Now we can conclude that the inequality (2.30) holds for all $n \in \mathbf{N}$. From (2.30), we obtain

$$
\lim _{n \rightarrow \infty} \Lambda^{n} \varepsilon_{m}(x)=0
$$

for all $x \in X_{0}$ and all $m \in U$. Hence, according to Theorem 1.4, there exists, for each $m \in U$, a unique solution $C_{m}: X_{0} \rightarrow Y$ of the equation

$C_{m}(x)=12 C_{m}(m x)+2 C_{m}((3 m-1) x)+2 C_{m}((-m+1) x)-C_{m}((4 m-1) x)$,

$$
x \in X_{0}
$$

such that

$$
\left\|f(x)-C_{m}(x)\right\|_{*} \leq \sup _{n \in \mathbf{N}_{0}}\left\{(\lambda(m)+\lambda(-2 m+1)) \alpha_{m}^{n} \varphi(x)\right\}, x \in X_{0} .
$$

Moreover,

$$
C_{m}(x):=\lim _{n \rightarrow \infty}\left(\mathcal{T}_{m}^{n} f\right)(x)
$$

for all $x \in X_{0}$. Now we show that 
$\left\|\mathcal{T}_{m}^{n} f(2 x+y)+\mathcal{T}_{m}^{n} f(2 x-y)-2 \mathcal{T}_{m}^{n} f(x+y)-2 \mathcal{T}_{m}^{n} f(x+y)-12 \mathcal{T}_{m}^{n} f(x)\right\|_{*}$

$$
\leq \alpha_{m}^{n}(\varphi(x)+\varphi(y))
$$

for every $x, y \in X_{0}$ such that $x+y \neq 0$ and $n \in \mathbf{N}$. Since the case $n=0$ is just (2.27), take $k \in \mathbf{N}$ and assume that (2.33) holds for $n=k$, where $k \in \mathbf{N}$ and every $x, y \in X_{0}$ such that $x+y \neq 0$. Then

$$
\begin{aligned}
& \left\|\mathcal{T}_{m}^{k+1} f(2 x+y)+\mathcal{T}_{m}^{k+1} f(2 x-y)-2 \mathcal{T}_{m}^{k+1} f(x+y)-2 \mathcal{T}_{m}^{k+1} f(x-y)-12 \mathcal{T}_{m}^{k+1} f(x)\right\|_{*} \\
& =\| 12 \mathcal{T}_{m}^{k} f(m(2 x+y))+2 \mathcal{T}_{m}^{k} f((3 m-1)(2 x+y))+2 \mathcal{T}_{m}^{k} f((-m+1)(2 x+y)) \\
& -\mathcal{T}_{m}^{k} f((4 m-1)(2 x+y))+12 \mathcal{T}_{m}^{k} f((m)(2 x-y))+2 \mathcal{T}_{m}^{k} f((3 m-1)(2 x-y)) \\
& +2 \mathcal{T}_{m}^{k} f((-m+1)(2 x-y))-\mathcal{T}_{m}^{k} f((4 m-1)(2 x-y))-24 \mathcal{T}_{m}^{k} f((m)(x+y)) \\
& -4 \mathcal{T}_{m}^{k} f((3 m-1)(x+y))-4 \mathcal{T}_{m}^{k} f((-m+1)(x+y))+2 \mathcal{T}_{m}^{k} f((4 m-1)(x+y)) \\
& -24 \mathcal{T}_{m}^{k} f((m)(x-y))-4 \mathcal{T}_{m}^{k} f\left(\left(\frac{3 m-1}{2}\right)(x-y)\right)-4 \mathcal{T}_{m}^{k} f((-m+1)(x-y)) \\
& +2 \mathcal{T}_{m}^{k} f((4 m-1)(x-y))-144 \mathcal{T}_{m}^{k} f((m)(x))-24 \mathcal{T}_{m}^{k} f\left(\left(\frac{3 m-1}{2}\right)(x)\right)- \\
& 24 \mathcal{T}_{m}^{k} f((-m+1)(x))+\left.12 \mathcal{T}_{m}^{k} f((4 m-1)(x))\right|_{*} \\
& \leq \max \left\{\| \mathcal{T}_{m}^{n} f(m(2 x+y))+\mathcal{T}_{m}^{n} f(m(2 x-y))-2 \mathcal{T}_{m}^{n} f(m(x+y))\right. \\
& -\mathcal{T}_{m}^{n} f(m(x-y))-12 \mathcal{T}_{m}^{n} f(m x)\left\|_{*},\right\| \mathcal{T}_{m}^{n} f((3 m-1)(2 x+y))+ \\
& \mathcal{T}_{m}^{n} f((3 m-1)(2 x-y))-2 \mathcal{T}_{m}^{n} f((3 m-1)(x+y))-\mathcal{T}_{m}^{n} f((3 m-1)(x-y))- \\
& 12 \mathcal{T}_{m}^{n} f((3 m-1) x)\left\|_{*},\right\| \mathcal{T}_{m}^{n} f((-m+1)(2 x+y))+\mathcal{T}_{m}^{n} f((-m+1)(2 x-y))- \\
& 2 \mathcal{T}_{m}^{n} f((-m+1)(x+y))-\mathcal{T}_{m}^{n} f((-m+1)(x-y))-12 \mathcal{T}_{m}^{n} f((-m+1) x) \|_{*} \\
& \| \mathcal{T}_{m}^{n} f((4 m-1)(2 x+y))+\mathcal{T}_{m}^{n} f((4 m-1)(2 x-y))-2 \mathcal{T}_{m}^{n} f((4 m-1)(x+y))- \\
& \left.\mathcal{T}_{m}^{n} f((4 m-1)(x-y))-12 \mathcal{T}_{m}^{n} f((4 m-11) x)_{*}\right\}
\end{aligned}
$$




$$
\begin{aligned}
& \leq \max \left\{\alpha _ { m } ^ { k } \left(\varphi\left((m x)+\varphi((m y)), \alpha_{m}^{k}(\varphi(3 m-1) x)+\varphi((3 m-1) y)\right)\right.\right. \\
& \left.\left.\alpha_{m}^{k}(\varphi(-m+1) x)+\varphi((-m+1) y)\right)\right) \\
& \left.\left.\alpha_{m}^{k}(\varphi(4 m-1) x)+\varphi((4 m-1) y)\right)\right\} \\
& \leq \alpha_{m}^{k} \max \{\lambda(m), \lambda(3 m-1), \lambda(-m+1), \lambda(4 m-1)\}(\varphi(x)+\varphi(y)) \\
& =\alpha_{m}^{k+1}(\varphi(x)+\varphi(y))
\end{aligned}
$$

Thus, by induction we have shown that (2.33) holds for every $n \in \mathbf{N}$. Letting $n \rightarrow \infty$ in (2.33), we obtain that

$$
C_{m}(2 x+y)+C_{m}(2 x-y)=2 C_{m}(x+y)+2 C_{m}(x-y)+12 C_{m}(x)
$$

for all $x, y \in X_{0}$ such that $x+y \neq 0$. In this way we obtain a sequence $\left\{C_{m}\right\}_{m \in U}$ of Cubic functions on $X_{0}$ such that

$$
\left\|f(x)-C_{m}(x)\right\|_{*} \leq \sup _{n \in \mathbf{N}_{0}}\left\{(\lambda(m+2)+\lambda(-m)) \alpha_{m}^{n} \varphi(x)\right\}, \quad x \in X_{0},
$$

this implies that

$$
\left\|f(x)-C_{m}(x)\right\|_{*} \leq(\lambda(m)+\lambda(-2 m+1)) \varphi(x), \quad x \in X_{0} .
$$

Because the precedent inequality holds for over $n=0$ and $\alpha_{m}<1$ 
It follows, with $m \rightarrow \infty$, that $f$ is Cubic on $X_{0}$.

The following corollary is a particular case of Theorem 2.5 where $\varphi(x):=$ $c\|x\|^{p}$ with $c \geq 0$ and $p<0$.

Corollary 2.6. Let $(X,\|\cdot\|)$ and $\left(Y,\|\cdot\|_{*}\right)$ be normed space and ultrametric Banach space respectively, $c \geq 0, p<0$ and let $f: X \rightarrow Y$ satisfy

$$
\begin{gathered}
\| f(2 x+y))+f(2 x+y)-2 f(x+y)-2 f(x-y)-12 f(x) \|_{*} \\
\leq c\left(\|x\|^{p}+\|y\|^{p}\right),
\end{gathered}
$$

for all $x, y \in X_{0}$. Then $f$ is Cubic on $X_{0}$.

\section{References}

[1] M. Almahalebi, A. Charifi and S. Kabbaj, Hyperstability of a monomial functional equation, Journal of Scientific Research Reports, 3 (20), pp. 2685-2693, (2014).

[2] M. Almahalebi and S. Kabbaj, Hyperstability of Cauchy-Jensen type functional equation, Advances in Research, 2 (12), pp. 1017-1025, (2014).

[3] M. Almahalebi and C. Park, On the hyperstablity of a functional equation in commutative groups, Journal of Computational Analysis and Applications, 20 (5) (2016), pp. 826-833, (2016).

[4] M. Almahalebi, A. Charifi and S. Kabbaj, Hyperstability of a Cauchy functional equation, Journal of Nonlinear Analysis and Optimization: Theory \& Applications, (In press).

[5] M. Almahalebi, On the hyperstability of $\sigma$-Drygas functional equation on semigroups, Aequationes math., 90 4, pp. 849-857, (2016).

[6] T. Aoki, On the stability of the linear transformation in Banach spaces, J. Math. Soc. Japan 2, pp. 64-66, (1950).

[7] A. Bahyrycz, J. Brzdȩk and M. Piszczek, On approximately p-Wright afine functions in ultrametric spaces, J. Funct. Spaces Appl., Art. ID 723545, (2013). 
[8] A. Bahyrycz and M. Piszczek, Hyperstability of the Jensen functional equation, Acta Math. Hungar., 142, pp. 353-365, (2014).

[9] A. Bahyrycz and J. Olko, Stability of the equation of $(p, q)$-Wright functions, Acta Math. Hung., 146, pp. 71-85, (2015).

[10] A. Bahyrycz and J. Olko, On stability of the general linear equation, Aequationes Math., 89, pp. 1461-1474, (2015).

[11] D. G. Bourgin, Approximately isometric and multiplicative transformations on continuous function rings, Duke Math. J. 16, pp. 385-397, (1949).

[12] J. Brzdȩk, J. Chudziak and Zs. Páles, A fixed point approach to stability of functional equations, Nonlinear Anal., 74, pp. 6728-6732, (2011).

[13] J. Brzdęk and K. Ciepliński, A fixed point approach to the stability of functional equations in non-Archimedean metric spaces, Nonlinear Analysis 74, pp. 6861-6867, (2011).

[14] J. Brzdȩk, Stability of additivity and fixed point methods, Fixed Point Theory and App., pp. 2013:285, 9 pages, (2013).

[15] J. Brzdęk, Hyperstability of the Cauchy equation on restricted domains, Acta Math. Hungar., 141, pp. 58-67, (2013).

[16] J. Brzdȩk, Remarks on hyperstability of the Cauchy functional equation, Aequationes Math., 86, pp. 255-267, (2013).

[17] J. Brzdęk, A hyperstability result for the Cauchy equation, Bull. Aust. Math. Soc., 89, pp. 33-40, (2014).

[18] J. Brzdęk and K. Ciepliński, Hyperstability and superstability, Abs. Appl. Anal., 2013, Article ID 401756, 13, (2013).

[19] J. Erdős, A remark on the paper On some functional equations by $S$. Kurepa, Glasnik Mat.-Fiz. Astronom., 14, pp. 3-5, (1959).

[20] P. Găvruţa, A generalization of the Hyers-Ulam-Rassias stability of approximately additive mappings, J. Math. Anal. Appl. 184, pp. 431436, (1994).

[21] E. Gselmann, Hyperstability of a functional equation, Acta Math. Hungar., 124, pp. 179-188, (2009). 
[22] D. H. Hyers, On the stability of the linear functional equation, Proc. Natl. Acad. Sci. U.S.A., 27, pp. 222-224, (1941).

[23] B. Jessen, J. Karpf and A. Thorup, Some functional equations in groups and rings, Math. Scand., 22, pp. 257-265, (1968).

[24] A. Khrennikov, Non-Archimedean Analysis: Quantum Paradoxes, Dynamical Systems and Biological Models., Kluwer Academic Publishers, Dordrecht, (1997).

[25] Gy. Maksa and Zs. Páles, Hyperstability of a class of linear functional equations, Acta Math., 17 (2), pp. 107-112, (2001).

[26] M. Piszczek, Remark on hyperstability of the general linear equation, Aequationes math., 88 (1), pp. 163-168, (2014).

[27] Th. M. Rassias, On the stability of the linear mapping in Banach spaces, Proc. Amer. Math. Soc., 72, pp. 297-300, (1978).

[28] M. Sirouni and S. Kabbaj, A fixed point approach to the hyperstability of Drygas functional equation in metric spaces. J. Math. Comput. Sci., 4 (4), pp. 705-715, (2014).

[29] S. M. Ulam, Problems in Modern Mathematics, Science Editions, JohnWiley \& Sons Inc. New York, (1964).

[30] D. Zhang, On Hyers-Ulam stability of generalized linear functional equation and its induced Hyers-Ulam programming problem, Aequationes Math., 90, pp. 559-568, (2016).

[31] D. Zhang, On hyperstability of generalised linear functional equations in several variables, Bull. Aust. Math. Soc., 92, pp. 259-267, (2015).

\author{
Youssef Aribou \\ Department of Mathematics, \\ Faculty of Sciences, \\ University of Ibn \\ Tofail, B. P. 133 Kenitra, \\ Morocco \\ e-mail : aribouyoussef3@gmail.com
}




\section{Muaadh Almahalebi}

Department of Mathematics, Faculty of Sciences, University of Ibn Tofail, B. P. 133 Kenitra, Morocco

e-mail : muaadh1979@hotmail.fr

and

\section{Samir kabbaj}

Department of Mathematics, Faculty of Sciences,

University of Ibn

Tofail, B. P. 133 Kenitra,

Morocco

e-mail : samkabbaj@yahoo.fr 